\title{
TOPOLOGICAL TRIVIALITY OF A FAMILY OF ZERO-SETS
}

\author{
MICHAEL A. BUCHNER AND WOJCIECH KUCHARZ
}

(Communicated by Haynes R. Miller)

\begin{abstract}
This paper gives conditions on a mapping $F: U \times \mathbf{R}^{k} \rightarrow \mathbf{K}^{p}$ ( $U \subset \mathbf{K}^{n}$ open, $\mathbf{K}=\mathbf{R}$ or $\mathbf{C}$ ) such that the family $F_{t}^{-1}(0)$ is a topologically trivial family (i.e., does not change topologically as $t \in \mathbf{R}^{k}$ varies). As an application an easy proof is given of a counterexample to a conjecture of Thom concerning the number of topologically different realizations of a given jet.
\end{abstract}

1. Introduction. Let $\mathbf{K}$ denote the field of real or complex numbers and let $U$ be a neighborhood of 0 in $\mathbf{K}^{n}$. Given a continuous map $F: U \times \mathbf{R}^{k} \rightarrow \mathbf{K}^{p}$ and $t$ in $\mathbf{R}^{k}$, we define $Z(F)=F^{-1}(0), F_{t}: U \rightarrow \mathbf{K}^{p}, F_{t}=F(\cdot, t)$ and $Z(F)_{t}=$ $F_{t}^{-1}(0)$. We think of $t, F$ and $Z(F)$ as parameter, family of maps and family of varieties, respectively. The goal of this note is to give sufficient conditions for $Z(F)$ (respectively $F$ ) to be locally topologically trivial at the origin. More precisely, given a point $t_{0}$ in $\mathbf{R}^{k}$ and a relatively compact neighborhood $V_{0}$ of $t_{0}$, we are interested in finding a neighborhood $U_{0}$ of 0 in $U$ and a continuous map $\sigma: U_{0} \times V_{0} \rightarrow$ $U$ such that for each $t$ in $V_{0}$ the map $\sigma_{t}=\sigma(\cdot, t)$ sends 0 onto 0 , homeomorphically transforms $U_{0}$ onto $\sigma_{t}\left(U_{0}\right), \sigma_{t}^{-1}\left(Z(F)_{t}\right)=U_{0} \cap Z(F)_{t_{0}}$ (respectively $F_{t} \circ \sigma_{t}=F_{t_{0}}$ on $\left.U_{0}\right)$ and $\sigma_{t_{0}}$ is the identity map from $U_{0}$ onto itself.

Even in the best understood case, for $p=1$ and under strong conditions imposed on $F$, the problem is far from solved. Assume that $F$ is of class $C^{\infty}$ and for each $t$ in $\mathbf{R}^{k}, F_{t}: U \rightarrow \mathbf{K}$ is a polynomial function having an isolated critical point at 0 . Then, by the results of Lê and Ramanujam [8] and Timourian [12], respectively, the families $Z(F)$ and $F$ are locally topologically trivial at the origin, provided $\mathbf{K}=\mathbf{C}$ and $n \neq 3$. The corresponding result holds in the real case, thanks to King's theorem [5], for $n \leq 3$ and is false for all $n \geq 5$. Clearly, in the real case, it is necessary to impose further restrictions on $F$. Following other authors, Damon [3, 4], Oka [9], we consider here families which are deformations of a quasihomogeneous polynomial map (cf. §2 for details).

Other papers related to this one are those of Buchner et al. [2] and Percell and Brown [10].

The main results are Theorems 2.3 and 2.5. $\S 2$ also contains some examples.

2. Deformations of quasi-homogeneous maps. Let $\alpha=\left(\alpha_{1}, \ldots, \alpha_{n}\right)$ be an $n$-tuple of positive integers and let $\gamma$ be a positive integer. A polynomial function $P: \mathbf{K}^{n} \rightarrow \mathbf{K}$ is said to be of type $(\alpha ; \gamma)$ if $P$ is a $\mathbf{K}$-linear combination of monomials $x_{1}^{i_{1}} \cdots x_{n}^{i_{n}}$ for which $\alpha_{1} i_{1}+\cdots+\alpha_{n} i_{n}=\gamma$. Given an analytic function-germ $f:\left(\mathbf{K}^{n}, 0\right) \rightarrow(\mathbf{K}, 0)$, we define $w(f, \alpha)$ to be the minimum of the

Received by the editors November 1, 1985 and, in revised form, October 20, 1986.

1980 Mathematics Subject Classification (1985 Revision). Primary 57D45, 58C25. 
integers $\alpha_{1} i_{1}+\cdots+\alpha_{n} i_{n}$ such that the monomial $x_{1}^{i_{1}} \cdots x_{n}^{i_{n}}$ appears in the Taylor expansion of $f$ at 0 with a nonzero coefficient. Now let $\alpha=\left(\alpha_{1}, \ldots, \alpha_{n}\right)$ and $\beta=\left(\beta_{1}, \ldots, \beta_{p}\right)$ be an $n$-tuple and a $p$-tuple of positive integers. A polynomial map $Q=\left(Q_{1}, \ldots, Q_{p}\right): \mathbf{K}^{n} \rightarrow \mathbf{K}^{p}$ is said to be of type $(\alpha ; \beta)$ if for each $j=1, \ldots, p$, $Q_{j}$ is of type $\left(\alpha ; \beta_{j}\right)$.

We need two more concepts.

DEFINITION 2.1. A polynomial map $Q: \mathbf{K}^{n} \rightarrow \mathbf{K}^{p}$ is said to be nondegenerate (resp. strongly nondegenerate) if for all $x$ in $Q^{-1}(0) \backslash\{0\}$ (resp. $\mathbf{K}^{n} \backslash\{0\}$ ) the derivative $d_{x} Q$ is surjective.

Note that if $Q^{-1}(0) \backslash\{0\} \neq \varnothing$, then nondegeneracy requires $n \geq p-1$.

DEFINITION 2.2. A map $F: U \times \mathbf{R}^{k} \rightarrow \mathbf{K}^{p}$, where $U$ is a neighborhood of 0 in $\mathbf{K}^{n}$, is called admissible (resp. strongly admissible) for $(\alpha ; \beta)$ if $F$ can be written as $F=Q+G$ with $Q, G: U \times \mathbf{R}^{k} \rightarrow \mathbf{K}^{p}$ satisfying the following conditions:

(i) the $j$ th components $Q_{j}$ and $G_{j}$ of $Q$ and $G$, resp., are of class $C^{2}, G_{j}$, $\partial G_{j} / \partial t_{s}, \partial^{2} G_{j} / \partial t_{s} \partial t_{q}, s, q=1, \ldots, k$, are of class $C^{r_{j}}$ in the $x$-variable, where $r_{j}>$ $\max \left\{\beta_{j} / \alpha_{1}, \ldots, \beta_{j} / \alpha_{n}\right\}$ and all derivatives in the $x$ variable up to order $r_{j}$ of $G_{j}$, $\partial G_{j} / \partial t_{s}, \partial^{2} G_{j} / \partial t_{s} \partial t_{q}$ are $C^{0}$ in $(x, t)$

(ii) for each $t$ in $\mathbf{R}^{k}, Q_{t}=Q(\cdot, t)$ is the restriction to $U$ of a nondegenerate (resp. strongly nondegenerate) map of type $(\alpha ; \beta)$ and, moreover, $G_{t}=G(\cdot, t)$ is holomorphic if $\mathbf{K}=\mathbf{C}$;

(iii) $w\left(T^{r_{j}-1} G_{j}(\cdot, t), \alpha\right)>\beta_{j}$ for all $t$ in $\mathbf{R}^{j}$ and $j=1, \ldots, p$, where $T^{r}(\cdot)$ denotes the Taylor polynomial (with respect to the variable in $\mathbf{K}^{n}$ ) at 0 of degree $r$. (Note that $w\left(T^{r_{j}} G_{j}(\cdot, t), \alpha\right)>\beta_{j}$ is also satisfied, since, by the inequality in (i), $w\left(x_{1}^{i_{1}} \cdots x_{n}^{i_{n}}\right)>\beta_{j}$ if $i_{1}+\cdots+i_{n} \geq r_{j}$.)

Our main result is the following.

THEOREM 2.3. Let $F: U \times \mathbf{R}^{k} \rightarrow \mathbf{K}^{p}$ be an admissible (resp. strongly admissible) map for $(\alpha ; \beta)$. Then given a point $t_{0}$ in $\mathbf{R}^{k}$ and a relatively compact neighborhood $V_{0}$ of $t_{0}$ in $\mathbf{R}^{k}$, there exists a neighborhood $U_{0}$ of 0 in $\mathbf{K}^{n}$ and a continuous map $\sigma: U_{0} \times V_{0} \rightarrow U$ such that for each $t$ in $V_{0}, \sigma_{t}$ sends 0 onto 0 , transforms homeomorphically $U_{0}$ onto $\sigma_{t}\left(U_{0}\right), \sigma_{t}^{-1}\left(F_{t}^{-1}(0)\right)=U_{0} \cap F_{t_{0}}^{-1}(0)\left(\right.$ resp. $F_{t} \circ \sigma_{t}=F_{t_{0}}$ on $\left.U_{0}\right)$ and $\sigma_{t_{0}}$ is the identity map from $U_{0}$ onto itself. In other words, the family of varieties $Z(F)$ (resp. the family of maps $F$ ) is locally topologically trivial at the origin.

Now we shall point out one application of Theorem 2.3. It is known that Thom's conjecture [11], stating that an $r$-jet $w$ in $J^{r}(n, p)$ admitting two topologically different realizations (i.e., for which one can find two map-germs $f, g:\left(\mathbf{R}^{n}, 0\right) \rightarrow$ $\left(\mathbf{R}^{p}, 0\right)$ whose $r$-jets are equal to $w$ and $g$ is always different from $\tau \circ f \circ \sigma$, where $\sigma:\left(\mathbf{R}^{n}, 0\right) \rightarrow\left(\mathbf{R}^{n}, 0\right)$ and $\tau:\left(\mathbf{R}^{p}, 0\right) \rightarrow\left(\mathbf{R}^{p}, 0\right)$ are local homeomorphisms $)$ has actually infinitely many topologically different realizations, is true if $p=1$ and $C^{r}$ or $C^{r+1}$ realizations are allowed [1]. The conjecture is false if we only allow $C^{r+2}$ realizations [7]. In the example that follows we show that the arguments of [7], involving the complexifications, computation of Milnor numbers and a delicate theorem of King [5], can be simplified by applying Theorem 2.3.

EXAMPLE 2.4. Consider the 6-jet $w=x_{1}^{3}-3 x_{1} x_{2}^{5}$ (we identify an $r$-jet with its unique polynomial realization of degree not exceeding $r)$. Let $f:\left(\mathbf{R}^{2}, 0\right) \rightarrow(\mathbf{R}, 0)$ be a $C^{8}$ function-germ whose 6 -jet is equal to $w$. We claim that there exists a local 
homeomorphism $\sigma:\left(\mathbf{R}^{2}, 0\right) \rightarrow\left(\mathbf{R}^{2}, 0\right)$ such that $f \circ \sigma=w+c x_{2}^{7}$ for some $c$ in $\mathbf{R}$ and a local homeomorphism $\sigma_{c}:\left(\mathbf{R}^{2}, 0\right) \rightarrow\left(\mathbf{R}^{2}, 0\right)$ for which $\left(w+c x_{2}^{7}\right) \circ \sigma_{c}=x_{1}^{3}+x_{2}^{7}$ if $c \neq 0$. Note that since the set-germs at 0 of $\left\{x \in \mathbf{R}^{2} \mid w(x)=0\right\}$ and $\{x \in$ $\left.\mathbf{R}^{2} \mid x_{1}^{3}+x_{2}^{7}=0\right\}$ are not homeomorphic, $w$ admits exactly two topologically different $C^{8}$ realizations. To prove the claim define $F(x, t)=w(x)+c x_{2}^{7}+t\left(f(x)-w(x)-c x_{2}^{7}\right)$, where $c$ is the coefficient of $x_{2}^{7}$ in the Taylor expansion of $f$ of order 7 . Note that $F$ is strongly admissible for $((5,2 ; 15)$ if $c=0$ (with $Q(x, t)=w(x)$ and $G(x, t)=t(f(x)-w(x)))$ and for $((7,3) ; 21)$ if $c \neq 0$ (with $Q(x, t)=x_{1}^{3}+c x_{2}^{7}$ and $\left.G(x, t)=-3 x_{1} x_{2}^{5}+t\left(f(x)-w(x)-c x_{2}^{7}\right)\right)$. In both cases there exists a local homeomorphism $\sigma:\left(\mathbf{R}^{2}, 0\right) \rightarrow\left(\mathbf{R}^{2}, 0\right)$ with $f \circ \sigma=F_{1} \circ \sigma=F_{0}=w+c x_{2}^{7}$. If $c \neq 0$, define $H(x, t)=x_{1}^{3}+c x_{2}^{7}-3 t x_{1} x_{2}^{7}$. Since $H$ is strongly admissible for $((7,3) ; 21)$ (with $Q(x, t)=x_{1}^{3}+c x_{2}^{7}$ and $\left.G(x, t)=-3 t x_{1} x_{2}^{5}\right)$, there exists a local homeomorphism $\tau_{c}:\left(\mathbf{R}^{2}, 0\right) \rightarrow\left(\mathbf{R}^{2}, 0\right)$ satisfying $\left(w+c x_{2}^{7}\right) \circ \tau_{c}=H_{1} \circ \tau_{c}=H_{0}=$ $x_{1}^{3}+c x_{2}^{7}$. It suffices to take $\sigma_{c}=\tau_{c} \circ \gamma$, where $\gamma(x)=\left(x_{1}, c^{-1 / 7} x_{2}\right)$.

Let $q$ and $m$ be nonnegative integers with $q+m=n$. Denote by $y$ and $\lambda$ variables in $\mathbf{K}^{q}$ and $\mathbf{K}^{m}$, respectively.

THEOREM 2.5. If condition (ii) in Definition 2.2 is replaced by

(ii') for each $t$ in $\mathbf{R}^{k}, Q_{t}$ is the restriction to $U$ of a polynomial map of type $(\alpha ; \beta)$, the partial derivative of $Q_{t}$ with respect to $y$ is surjective for all $(y, \lambda)$ in $Q_{t}^{-1}(0) \backslash\{0\}$ (resp. $\mathbf{K}^{n} \backslash\{0\}$ ) and, moreover, $G_{t}$ is holomorphic if $\mathbf{K}=\mathbf{C}$,

then under the assumptions of Theorem 2.3 each homeomorphism $\sigma_{i}$ is of the form $\sigma_{t}(y, \lambda)=\left(\bar{\sigma}_{t}(y, \lambda), \lambda\right)$.

After giving the proof of 2.3 we will explain the modifications needed to prove 2.5 .

The following example shows that a result in [10] can be inproved upon by application of Theorem 2.5.

EXAMPLE $2.6[10]$. Let $f\left(y_{1}, y_{2}, \lambda\right)=\left(y_{2}+\lambda y_{1}, y_{1}^{2}+\lambda y_{2}\right)$ and $g=f+h$, where $h=\left(h_{1}, h_{2}\right), h_{1}$ is of class $C^{4}$ and $h_{2}$ is of class $C^{5}$. Assume that $w\left(T^{3} h_{1},(2,3,1)\right)>$ 3 and $w\left(T^{4} h_{2},(2,3,1)\right)>4$. Then there exists a local homeomorphism $\sigma:\left(\mathbf{R}^{2} \times\right.$ $\mathbf{R}, 0) \rightarrow\left(\mathbf{R}^{2} \times \mathbf{R}, 0\right)$ of the form $\sigma(y, \lambda)=(\bar{\sigma}(y, \lambda), \lambda)$ such that $f^{-1}(0)=\sigma\left(g^{-1}(0)\right)$. To show this it suffices to apply Theorem 2.5 to the family $F(y, \lambda, t)=f(y, \lambda)+$ $\operatorname{th}(y, \lambda)$ which is admissible for $((2,3,1) ;(3,4))$ (with (ii') in Definition 2.2 instead of (ii)). Note that [10] gives the same conclusion under the stronger assumptions $T^{3} h_{1}=0$ and $T^{4} h_{2}=0$.

3. The proof of Theorem 2.3. This section is devoted to proving Theorem 2.3. Clearly, the complex case follows from the real one, so we assume $\mathbf{K}=\mathbf{R}$. Without loss of generality we may assume that $t_{0}=0$ and that $V_{0}=$ the cube $(-L, L)^{k}$ for some positive real number $L$. Let $S^{n-1}$ be the unit sphere in $\mathbf{R}^{n}$ and let $\varepsilon>0$ be chosen in such a way that the map $\phi: S^{n-1} \times(-\varepsilon, \varepsilon) \rightarrow \mathbf{R}^{n}$ defined by

$$
\psi\left(x_{1}, \ldots, x_{n}, r\right)=\left(r^{\alpha_{1}} x_{1}, \ldots, r^{\alpha_{n}} x_{n}\right)
$$

has values in $U$.

For each $j=1, \ldots, p$ define $H_{j}: S^{n-1} \times(-\varepsilon, \varepsilon) \times \mathbf{R}^{k} \rightarrow \mathbf{R}$ by $H_{j}(x, r, t)=$ $r^{-\beta_{j}} G_{j}(\phi(x, r), t)$ for $r \neq 0$ and $H_{j}(x, 0, t)=0$. Conditions (i) and (iii) of Definition 2.2 imply that $H_{j}, \partial H_{j} / \partial x_{l}$ and $\partial H_{j} / \partial t_{s}, l=1, \ldots, n, s=1, \ldots, k$, are at least of class $C^{1}$ and are equal to 0 if $r=0$ (this is obvious if $G_{j}$ is of class $C^{\infty}$, for 
then $H_{j}, \partial H_{j} / \partial x_{l}$ and $\partial H_{j} / \partial t_{s}$ are also of class $C^{\infty}$ and it requires a lengthy but straightforward computation if $G_{j}$ merely satisfies the differentiability conditions (i)). Let $E_{i j}$ be the $p \times p$ matrix whose entry in the $i$ th row and $j$ th column is equal to 1 and all other entries are equal to 0 . Let $\pi: S^{n-1} \times(-\varepsilon, \varepsilon) \times \mathbf{R}^{k} \rightarrow S^{n-1} \times \mathbf{R}^{k}$ be the projection and let $A$ be the $p \times\left(p^{2}+n\right)$ matrix whose columns consist of the columns $E_{i j} \cdot(Q \circ \pi+H)$ (product of $p \times p$ matrix by $p \times 1$ matrix) and the columns of the $p \times n$ matrix $C=\left(\left(\partial Q_{j} / \partial x_{l}\right) \circ \pi+\partial H_{j} / \partial x_{l}\right)$. Finally we denote by $A^{*}$ the matrix whose columns consist of the columns $E_{i j} \cdot(Q \circ \pi)$ and the columns of $C^{*}=\left(\left(\partial Q_{j} / \partial x_{l}\right) \circ \pi\right)$. (In the above, $i, j=1, \ldots, p$ and $l=1, \ldots, n$.)

Note that all entries of $A$ are $C^{1}$ functions in the variables $(x, r, t)$. By the nondegeneracy condition, $A^{*}$ has rank $p$ at each point in $S^{n-1} \times(-\varepsilon, \varepsilon) \times \mathbf{R}^{k}$ and hence $A$ has the same property when $\varepsilon$ is chosen suitably small and $\mathbf{R}^{k}$ is replaced by a compact set which we take to be $[-L-\delta, L+\delta]^{k}$. It follows that the column $\left(\left(\partial Q / \partial t_{k}\right) \circ \pi+\left(\partial H / \partial t_{k}\right)\right)$ is a linear combination of the columns of the matrix $A$ with coefficients which are $C^{1}$ functions on $S^{n-1} \times(-\varepsilon, \varepsilon) \times(-L-\delta, L+\delta)^{k}$. Thus

$$
\frac{\partial Q}{\partial t_{k}} \circ \pi+\frac{\partial H}{\partial t_{k}}=B \cdot\left(Q \circ \pi_{\bullet}+H\right)+C \cdot u
$$

where $B$ is a $p \times p$ matrix with $C^{1}$ entries and $u$ is an $n$-vector with $C^{1}$ components $u_{1}, \ldots, u_{n}$. Note that if $F$ is strongly admissible, then, by the same argument as above, the matrix $C$ has rank $p$ at each point in $S^{n-1} \times(-\varepsilon, \varepsilon) \times[-L-\delta, L+\delta]$ and we may assume that $B$ is the zero matrix. Now multiply the $i$ th component of (3.1) by $r^{\beta_{i}}$. By definition of $H_{j}$,

$$
\frac{\partial F}{\partial t_{k}}(\psi(x, r), t)=\tilde{B}(x, r, t) \cdot F(\psi(x, r), t)+d_{\psi(x, r)} F_{t}(w(x, r, t))
$$

on $S^{n-1} \times((-\varepsilon, 0) \cup(0, \varepsilon)) \times(-L-\delta, L+\delta)^{k}$ where $\tilde{B}(x, r, t)$ is a $p \times p$ matrix with entries which are $C^{1}$ on $S^{n-1} \times((-\varepsilon, 0) \cup(0, \varepsilon)) \times(-L-\delta, L+\delta)^{k}$ and where the $i$ th component $w_{i}(x, r, t)$ of $w(x, r, t)$ is equal to $r^{\alpha_{i}} u_{i}(x, r, t)$ which is $C^{1}$ on $S^{n-1} \times(-\varepsilon, \varepsilon) \times(-L-\delta, L+\delta)^{k}$.

Now (3.2) can be rewritten as

$$
\frac{\partial F}{\partial t_{k}}(x, t)=\tilde{B}\left(\psi^{-1}(x), t\right) F(x, t)+d_{x} F_{t}\left(w\left(\psi^{-1}(x), t\right)\right)
$$

where $x \in \psi\left(S^{n-1} \times(-\varepsilon, \varepsilon)\right) \backslash\{0\}$. We can regard $w\left(\psi^{-1}(x), t\right)$ as a vector field on $\psi\left(S^{n-1} \times(-\varepsilon, \varepsilon)\right) \backslash\{0\}$. We choose to regard it as a time dependent vector field; with $t_{k}$ being the time variable and $\left(t_{1}, \ldots, t_{k-1}\right)$ being a parameter. We wish to integrate this vector field, i.e. solve

$$
\left\{\begin{array}{l}
\partial \tau^{k}(x, t) / \partial t_{k}=-w_{t} \circ \psi^{-1}\left(\tau^{k}(x, t)\right) \\
\tau^{k}\left(x, t_{1}, \ldots, t_{k-1}, 0\right)=x
\end{array}\right.
$$

Suppose, for the moment, that $\varepsilon_{k}, \delta_{k}$ and $\tau_{k}$ can be found such that $0<\varepsilon_{k}<\varepsilon$, $0<\delta_{k}<\delta$ and $\tau^{k}$ is a mapping from

$$
\left(\psi\left(S^{n-1} \times\left(-\varepsilon_{k}, \varepsilon_{k}\right)\right) \backslash\{0\}\right) \times\left(-L-\delta_{k}, L+\delta_{k}\right)^{k} \rightarrow \psi\left(S^{n-1} \times(-\varepsilon, \varepsilon)\right) \backslash\{0\}
$$

satisfying (3.4). Let

$$
T^{k}:\left(\psi\left(S^{n-1} \times\left(-\varepsilon_{k}, \varepsilon_{k}\right)\right) \backslash\{0\}\right) \times\left(-L-\delta_{k}, L+\delta_{k}\right)^{k} \rightarrow G L(p, \mathbf{R})
$$


be the solution to

$$
\left\{\begin{array}{l}
\partial T^{k}(x, t) / \partial t_{k}=-T^{k}(x, t) \cdot \tilde{B}\left(\psi^{-1}\left(\tau^{k}(x, t)\right), t\right) \\
T^{k}\left(x, t_{1}, \ldots, t_{k-1}, 0\right)=E(\text { identity } p \times p \text { matrix }) .
\end{array}\right.
$$

The equations (3.3), (3.4) and (3.5) together imply

$$
\frac{\partial}{\partial t_{k}}\left(T^{k}(x, t) \cdot F\left(\tau^{k}(x, t), t\right)\right)=0
$$

from which it follows that

$$
T^{k}(x, t) \cdot F\left(\tau^{k}(x, t), t\right)=F\left(x, t_{1}, \ldots, t_{k-1}, 0\right) .
$$

In case $F$ is strongly admissible, $T^{k}(x, t)$ can be taken to be $E$ because in that case $B$, and hence $\tilde{B}$, can be taken to be the zero matrix.

It will also be shown, in the course of the construction of $\tau^{k}(x, t)$, that if $\tau^{k}(0, t)$ is defined to be 0 then $\tau_{t}^{k}: \psi\left(S^{n-1} \times\left(-\varepsilon_{k}, \varepsilon_{k}\right)\right) \rightarrow \psi\left(S^{n-1} \times(-\varepsilon, \varepsilon)\right)$ is a homeomorphism.

Granted this, (2.3) follows by induction with respect to the number of parameters.

To prove the claims made about $\tau^{k}$ it is convenient to lift the vector field $w_{t} \circ \psi^{-1}$ to $S^{n-1} \times((-\varepsilon, 0) \cup(0, \varepsilon))$. It will be shown that the lifted vector field has a $C^{1}$ extension to $S^{n-1} \times(-\varepsilon, \varepsilon)$ which is tangent to $S^{n-1} \times\{0\}$ at all points of $S^{n-1} \times\{0\}$. Assume this has been done. Denote the lifted vector field by $\xi_{t}$. Consider solving the equations

$$
\left\{\begin{array}{l}
\partial \tilde{\tau}^{k}(x, r, t) / \partial t_{k}=-\xi_{t}\left(\tilde{\tau}^{k}(x, r, t)\right) \\
\tilde{\tau}^{k}\left(x, r, t_{1}, \ldots, t_{k-1}, 0\right)=(x, r)
\end{array}\right.
$$

This has a $C^{1}$ solution on $S^{n-1} \times\left(-\varepsilon_{k}, \varepsilon_{k}\right) \times\left(-L-\delta_{k}, L+\delta_{k}\right)^{k}$ for suitable $0<\varepsilon_{k}<\varepsilon$ and $0<\delta_{k}<\delta$. Furthermore, $\tilde{\tau}_{t}^{k}$ is a $C^{1}$ embedding $S^{n-1} \times\left(-\varepsilon_{k}, \varepsilon_{k}\right) \rightarrow$ $S^{n-1} \times(-\varepsilon, \varepsilon)$ such that

$$
\tilde{\tau}_{t}^{k}\left(S^{n-1} \times\{0\}\right)=S^{n-1} \times\{0\}, \quad \tilde{\tau}_{t}^{k}\left(S^{n-1} \times\left(0, \varepsilon_{k},\right)\right) \subset S^{n-1} \times(0, \varepsilon)
$$

and

$$
\tilde{\tau}_{t}^{k}\left(S^{n-1} \times\left(-\varepsilon_{k}, 0\right)\right) \subset S^{n-1} \times(-\varepsilon, 0) .
$$

If we define $\tau_{t}^{k}$ by $\tau_{t}^{k}=\psi \circ \tilde{\tau}_{t}^{k} \circ \psi^{-1}$ on $\psi\left(S^{n-1} \times\left(0, \varepsilon_{k}\right)\right)$ then $\tau_{t}^{k}$ satisfies (3.4) and has all the desired properties.

Only the $C^{1}$ extension of the lifting remains to be shown. Recall that

$$
w_{t}(x, r)=\left(r^{\alpha_{1}} u_{1}(x, r, t), \ldots, r^{\alpha_{n}} u_{n}(x, r, t)\right)
$$

where each $u_{i}$ is $C^{1}$ on $S^{n-1} \times(-\varepsilon, \varepsilon) \times(-L-\delta, L+\delta)$. The vector field $\xi_{t}$ is the solution of $d_{(x, r)} \psi\left(\xi_{t}\right)=w_{t}(x, r)$. If we identify the tangent bundle of $S^{n-1}$ with a subbundle of the trivial bundle $S^{n-1} \times \mathbf{R}^{n}$ then the equation for $\xi_{t}$ becomes

$$
\left[\begin{array}{ccccc}
r^{\alpha_{1}} & 0 & \ldots & 0 & \alpha_{1} x_{1} r^{\alpha_{1}-1} \\
\vdots & \vdots & & \vdots & \vdots \\
0 & 0 & \ldots & r^{\alpha_{n}} & \alpha_{n} x_{n} r^{\alpha_{n}-1} \\
x_{1} & x_{2} & \ldots & x_{n} & 0
\end{array}\right]\left[\begin{array}{c}
\xi_{1 t} \\
\vdots \\
\xi_{n t} \\
\xi_{(n+1) t}
\end{array}\right]=\left[\begin{array}{c}
r^{\alpha_{1}} u_{1}(x, r, t) \\
\vdots \\
r^{\alpha_{n}} u_{n}(x, r, t) \\
0
\end{array}\right]
$$

In solving for each $\xi_{i t}$ by Cramer's rule we see that the denominator is the product of $r^{\left(\sum_{i} \alpha_{i}\right)-1}$ and a nonvanishing term and the numerator has a factor of $r^{\left(\sum_{i} \alpha_{i}\right)-1}$. 
This proves that $\xi_{t}$ has the claimed $C^{1}$ extension. Finally we observe that in using Cramer's rule for $\xi_{n+1}$ we obtain a factor of $r \sum_{i} \alpha_{i}$ in the numerator, thus justifying the claim that the extension is tangent to $S^{n-1} \times\{0\}$.

4. The proof of Theorem 2.5. We proceed exactly as in the proof of 2.3 except that we make the following additional observtion: The $p \times\left(p^{2}+q\right)$ matrix whose columns consist of the columns $E_{i j} \cdot(Q \circ \pi)$ and the columns of $\left(\left(\partial Q_{j} / \partial y_{l}\right) \circ \pi\right)$ $(i, j=1, \ldots, p$ and $l=1, \ldots, q)$ has rank $p$. Hence we will again obtain (3.1) but can assume $u_{q+1}, \ldots, u_{n}$ are all identically zero. When we solve (3.4) (with $x=(y, \lambda)$ ), we will find the last $m$ components of $\tau^{k}$ are constants and must therefore be $\lambda$, by the assumption of the initial condition.

5. Alternative proof of 2.3 suggested by referee. We now outline a second approach to Theorem 2.3 suggested by the referee and using the papers [5 and $\mathbf{6}$ ] by H. King. We consider $F(\phi(x, r), t)=F\left(r^{\alpha_{1}} x_{1}, \ldots, r^{\alpha_{n}} x_{n}, t\right)$ with $x=\left(x_{1}, \ldots, x_{n}\right) \in$ $S^{n-1}$ and $r \geq 0$. By (iii) we can write

$$
\begin{aligned}
& F\left(r^{\alpha_{1}} x_{1}, \ldots, r^{\alpha_{n}} x_{n}, t\right) \\
& \quad=\left(r^{\beta_{1}} Q_{1}(x, t)+r^{\beta_{1}+1} \tilde{G}_{1}(x, r, t), \ldots, r^{\dot{\beta}_{n}} Q_{n}(x, t)+r^{\beta_{n}+1} \tilde{G}_{n}(x, r, t)\right)
\end{aligned}
$$

where $\tilde{G}_{j}(x, r, t)$ is $C^{r_{j}}$ on $S^{n-1} \times(0, \infty)$ for each $t$ and $C^{0}$ on $S^{n-1} \times[0, \infty) \times \mathbf{R}^{k}$. It follows that if $t \in V_{0}, F(\phi(x, r), t)=0$ and $r$ is small then $x$ is close on $S^{n-1}$ to a zero of $Q_{t}$. Now, from (ii) we have $d_{(x, r)}\left(Q_{t} \circ \phi\right): T_{(x, r)}\left(S^{n-1} \times(0, \infty)\right) \rightarrow \mathbf{R}^{p}$ is surjective if $\left(Q_{t} \circ \phi\right)(x, r)=0$. From this and the quasihomogeneity of $Q_{t}$, it follows that $d_{x} Q_{t}: T_{x} S^{n-1} \rightarrow \mathbf{R}^{p}$ is surjective whenever $Q_{t}(x)=0$. Hence, for small $r$, we have $d_{x} Q_{t}$ is surjective whenever $t \in V_{0}$ and $F(\phi(x, r), t)=0$. Next, we compute

$$
d_{x}\left(F_{t} \circ \phi_{r}\right)=\left(r^{\beta_{1}} d_{x} Q_{1, t}+r^{\beta_{1}+1} d_{x} \tilde{G}_{1, r, t}, \ldots, r^{\beta_{n}} d_{x} Q_{n, t}+r^{\beta_{n}+1} d_{x} \tilde{G}_{n, r, t}\right) .
$$

Now it follows from the assumptions that $d_{x} \tilde{G}_{j, r, t}$ extends continuously to $r=0$ and hence is bounded for $r$ small, $t \in V_{0}$ and $x \in S^{n-1}$. We conclude that $d_{x}\left(F_{t} \circ \phi_{r}\right)$ is surjective for $r$ small, $t \in V_{0}$ and $x \in S^{n-1}$ and $F_{t} \circ \phi_{r}(x)=0$. This shows that $\left\{(x, r) \in S^{n-1} \times(0, \infty) \mid F_{t} \circ \phi_{r}(x)=0\right\}$ is a submanifold of $S^{n-1} \times(0, \infty)$ when $r$ is small and $t \in V_{0}$ and this submanifold is transverse to each $S^{n-1} \times\{r\}$.

In $\mathbf{R}^{n}$ the above argument shows the following for the mapping $F$ : There is a neighborhood of 0 in $\mathbf{R}^{n}$ of the form $U=\left\{\left(x_{1}, \ldots, x_{n}\right) \mid\left(x_{1} / \varepsilon^{\alpha_{1}}\right)^{2}+\cdots+\right.$ $\left.\left(x_{n} / \varepsilon^{\alpha_{n}}\right)^{2}<1\right\}$ such that for every $t \in V_{0},\left(F_{t}^{-1}(0) \cap U\right) \backslash\{0\}$ is a submanifold of $\mathbf{R}^{n} \backslash\{0\}$ transversal to every ellipsoid of the form $\left\{\left(x_{1}, \ldots, x_{n}\right) \mid\left(x_{1} / r^{\alpha_{1}}\right)^{2}+\cdots+\right.$ $\left.\left(x_{n} / r^{\alpha_{n}}\right)^{2}=1\right\}$ for $r<\varepsilon$ and such that $\left(F^{-1}(0) \cap\left(U \times V_{0}\right)\right) \backslash\{0\} \times V_{0}$ is a $C^{2}$ submanifold.

Let now $r\left(x_{1}, \ldots, x_{n}\right)$ be the $r$-component of the inverse of $\phi\left(x_{1}, \ldots, x_{n}, r\right)=$ $\left(r^{\alpha_{1}} x_{1}, \ldots, r^{\alpha_{n}} x_{n}\right)$. The transversality assertion is equivalent to the assertion that $r$ restricted to $\left(F_{t}^{-1}(0) \cap U\right) \backslash\{0\}$ has no critical points. Consequently, the map $(U \backslash\{0\}) \times V_{0} \rightarrow(0, \varepsilon) \times V_{0}$ given by $(x, t) \rightarrow(r(x), t)$ is a $C^{2}$ submersion which restricts to a $C^{2}$ submersion from $\left(F^{-1}(0) \cap\left(U \times V_{0}\right)\right) \backslash\{0\} \times V_{0}$ to $(0, \varepsilon) \times V_{0}$. Since the submersion is also proper it follows from the Ehresmann fibration theorem that $(U \backslash\{0\}) \times V_{0}$ is a locally trivial fibration over $(0, \varepsilon) \times V_{0}$ in such a way that $\left(F^{-1}(0) \cap\left(U \times V_{0}\right)\right) \backslash\{0\} \times V_{0}$ is a sublocally-trivial fibration over $(0, \varepsilon) \times V_{0}$. There is no loss in generality in assuming $V_{0}$ is contractible. Hence, these fibrations are actually topologically trivial. Choose $0<r_{0}<\varepsilon$ and let $E_{r_{0}}$ be the ellipsoid 
$\left\{\left(x_{1}, \ldots, x_{n}\right) \mid \sum_{i}\left(x_{i} / r_{0}^{\alpha_{i}}\right)^{2}=1\right\}$. There is a homeomorphism $\phi:(U \backslash\{0\}) \times V_{0} \rightarrow$ $(0, \varepsilon) \times E_{r_{0}} \times V_{0}$ of the form $(x, t) \rightarrow(r(x), \xi(x, t), t)$ which restricts to a homeomoprhism

$$
F^{-1}(0) \cap\left(U \times V_{0}\right) \backslash\{0\} \times V_{0} \rightarrow(0, \varepsilon) \times\left(F_{t_{0}}^{-1}(0) \cap E_{r_{0}}\right) \times V_{0} .
$$

So, the mapping $\gamma_{t}: x \mapsto\left(r(x), \xi_{t}(x)\right)$ is a homeomorphism $U \backslash\{0\} \rightarrow(0, \varepsilon) \times E_{r_{0}}$ mapping $\left(F_{t}^{-1}(0) \cap U\right) \backslash\{0\}$ onto $(0, \varepsilon) \times F_{t_{0}}^{-1}(0) \cap E_{r_{0}}$. It suffices now to define $\sigma_{t}$ by $\gamma_{t}^{-1} \circ \gamma_{t_{0}}$ on $U \backslash\{0\}$ and $\sigma_{t}(0)=0$.

The above argument proves the admissible version of 2.3. The strongly admissible version now follows by applying Theorem 1 of the paper [5] by $\mathrm{H}$. King, which gives a criterion (easily seen to be satisfied in our case) for when topological triviality of the family of germs of zero sets is equivalent to topological triviality of the family of germs of the mappings.

It is not clear to us if it is possible to modify the above procedure to give a proof of 2.5 .

\section{REFERENCES}

1. J. Bochnak and T. C. Kuo, Different realizations of a nonsufficient jet, Indag. Math. 34 (1972), 24-31.

2. M. Buchner, J. Marsden and S. Schecter, Applications of the blowing-up construction and algebraic geometry to bifurcation problems, J. Differential Equations 48 (1983), 404-432.

3. J. Damon, Finite determinacy and topological triviality, Invent. Math. 62 (1980), 299-324.

4. - Newton filtrations, monomial algebras and nonisolated and equivariant singularities, Proc. Sympos. Pure Math., vol. 40, Amer. Math. Soc., Providence, R.I., 1983, pp. 267-276.

5. H. King, Topological type in families of germs, Invent. Math. 62 (1980), 1-13.

6. _ Topological type of isolated critical points, Ann. of Math. (2) 107 (1978), 385-397.

7. S. Koike and W. Kucharz, Sur les réalisations de jets nonsuffisants, C. R. Acad. Sci. Paris Sér. A 288 (1979), 457-459.

8. D. T. Lê and C. P. Ramanujam, The invariance of Milnor's number implies the invariance of topological type, Amer. J. Math. 98 (1976), 67-78.

9. M. Oka, On the topology of the Newton boundary. II, J. Math. Soc. Japan 32 (1980), 65-92.

10. P. B. Percell and P. N. Brown, Finite determination of bifurcation problems, SIAM J. Math. Anal. 16 (1985), 28-46.

11. R. Thom, Problems in: Manifolds-Amsterdam 1970, Lecture Notes in Math., vol. 197 (N. Kuiper, ed.), Springer-Verlag, Berlin, 1971.

12. J. G. Timourian, The invariance of Milnor's number implies topological triviality, Amer. J. Math. 99 (1977), 437-466.

Department of Mathematics and Statistics, University of New Mexico, Albuquerque, NeW MEXICO 87131 